Check for updates

Cite this: RSC Adv., 2017, 7, 27272

Received 8th March 2017

Accepted 24th April 2017

DOI: $10.1039 / \mathrm{c} 7 \mathrm{ra02815f}$

rsc.li/rsc-advances

\section{Chirality-mediated enhancement of nitric oxide release and regulation of endothelial cells behaviors by cystine immobilization on $\mathrm{Ti}-\mathrm{O}$ films $\uparrow$}

\author{
Yajun Weng, (D) *ab Sisi Wu, ab Yonghong Fan, ${ }^{\text {ab }}$ Honghong Han, ${ }^{\text {ab }}$ Hong Wang ${ }^{\text {ab }}$ \\ and Nan Huang ${ }^{\text {ab }}$
}

Endogenous nitric oxide (NO), generated by endothelial cells (ECS), plays a critical role in the cardiovascular system. However, the effect of biomaterial-induced NO release on ECs is not clear. In this study, cystine with different chirality was immobilized on $\mathrm{Ti}-\mathrm{O}$ films to catalyze endogenous S-nitrosothiol decomposition to generate NO. Chemiluminescence analysis showed that a stable, sustained release of $\mathrm{NO}$ at a speed similar to that in healthy ECs was achieved on both enantiomer immobilized surfaces. However, L-cystine-immobilized surfaces induced higher NO release than D-cystine-immobilized surfaces. Although BSA adsorption was enhanced on L-surfaces, according to QCM analysis, preadsorption of BSA on L-surface still had a significantly higher NO release than that on the D-surface, indicating that the adsorption of BSA on L-surfaces was reversible. Platelet activation on the L-surfaces was obviously inhibited because of induction of more NO release. The growth, migration, and NO secretion behaviors of ECs were promoted by increased $N O$ release on the L-surfaces. These results show that L-cystine-immobilized surfaces are beneficial for the induction of NO release and regulation of the behaviors of ECs, providing a promising method for the endothelialization of vascular biomaterials.

\section{Introduction}

Constitutive release of NO by endothelial cells plays a critical role in maintaining blood vessel patency and inhibiting platelet aggregation and smooth muscle proliferation..$^{1-3}$ Many recent studies have reported that localized NO release is an effective method for improving the hemocompatibility of bloodcontacting medical devices. ${ }^{4,5}$ In our previous study, small molecules of disulfide or diselenide were immobilized on cardiovascular biomaterials to catalyze endogenous $S$-nitrosothiol decomposition to locally generate $\mathrm{NO}^{6-8}$ All these modified biomaterials showed improved properties of inhibition of platelet activation and smooth muscle proliferation. However, there were some contradictory results when the effects of NO on the EC behavior were explored in the literature. Some studies showed that NO plays an important role in mediating angiogenesis by supporting the migration and survival of ECs, ${ }^{9,10}$ whereas other studies showed that addition of NO donor inhibits the proliferation and migration of ECs. ${ }^{11,12}$ These

${ }^{a}$ Key Laboratory of Advanced Technologies of Materials, Ministry of Education,
Southwest Jiaotong University, China. E-mail: wengyj7032@swjtu.edu.cn; Fax: +86
28 87600625; Tel: +862887634148
${ }^{b}$ School of Materials Science and Engineering, Southwest Jiaotong University, Chengdu
610031 , China

$\dagger$ Electronic supplementary information (ESI) available. See DOI: $10.1039 / \mathrm{c} 7 \mathrm{ra} 02815 \mathrm{f}$ reports suggest that NO can act as an important protective molecule for ECs, as well as a cytotoxic agent, depending on the microenvironmental conditions.

Endogenous NO production is considered as the critical function of ECs. Healthy ECs continuously secrete NO at a steady speed of $0.5-4.0 \times 10^{-10} \mathrm{~mol} \mathrm{~cm}^{-2} \mathrm{~min}^{-1}$, and when ECs are damaged, NO production decreases..$^{13}$ It is reasonable to hypothesize that NO release at a steady speed in the ECs may not be cytotoxic to ECs; however, it is not sure whether higher speed of NO release is better. Since NO has a half time of a few seconds and then it transforms into nitrite/nitrate, the Griess reagent colorimetric method is usually employed to measure NO production by ECs. Herein, we used the chemiluminescence method to measure the concentration of nitrite/nitrate, resulting from the NO production by ECs; this method is more sensitive. Moreover, nearly all the abovementioned studies were based on NO donor or NO donor-loaded biomaterials. These NO donor or NO donor-loaded biomaterials usually have a burst NO release and cannot sustain a steady release.

Some recent studies have shown that surfaces with different chirality may trigger different interface biological process and cell-material interactions. Wang et al. ${ }^{14}$ found that $\mathrm{L}$-valine and D-valine-grafted polymer showed different protein adsorption behavior, and Bandyopadhyay et al. ${ }^{15}$ reported that chiral monolayers of polyol-terminated alkanethiols exhibited different resistances to protein adsorption and bacterial biofilm formation. It has also been reported that stem-cell 
differentiation behavior varies on different chiral surfaces. ${ }^{16} \mathrm{We}$ recently reported that L-selenocystine-immobilized surfaces showed higher catalytic activity in the decomposition of $S$ nitrosoglutathione (GSNO) in the presence of glutathione (GSH) and could improve hemocompatibility. ${ }^{17}$ In the present study, two enantiomers of cystine were immobilized on Ti-O films, and their influences on the catalytic NO release and EC behavior were studied.

\section{Materials and methods}

\subsection{Materials}

3,4-Dihydroxyphenylalanine (dopamine), L-cystine, D-cystine, $S$ nitrosoglutathione (GSNO), L-glutathione (GSH), ethylenediaminetetraacetic acid disodium salt (EDTA- $\left.\mathrm{Na}_{2}\right)$, bovine serum albumin (BSA), and cell counting kit-8 were purchased from Sigma-Aldrich Chemical Co. Other chemicals were of analytically pure grade and were purchased from Jin Shan Chemicals. Phosphate buffered saline (PBS, $0.01 \mathrm{M}, \mathrm{pH}=7.4$ ) was used in the NO release experiments. Milli-Q-water $(R=18.2$ $\mathrm{M} \Omega$ ) was used for other experiments.

\subsection{Preparation of samples}

Ti-O film-deposited silicon wafers were prepared by unbalanced magnetron sputtering ${ }^{18}$ and used as the substrate. It's mainly anatase phase of $\mathrm{TiO}_{2}$ and the thickness of the film is about $300 \mathrm{~nm}$. The as-deposited Ti-O on Si wafers was cut into 8 $\mathrm{mm} \times 8 \mathrm{~mm}$ plates for all the samples, whereas the samples for NO release were cut into $8 \mathrm{~mm} \times 4 \mathrm{~mm}$. The substrates were sonicated in acetone, ethanol, and water in sequence each step 3 times. All the cleaned Ti-O substrates were placed into a 24well plate. Then, five-layered polydopamine was prepared as described in the literature. ${ }^{7}$ Briefly, a first layer of polydopamine was grafted on the Ti-O surface via immersing the substrates in a $0.5 \mathrm{~mL}$ solution of $2 \mathrm{mg} \mathrm{mL}^{-1}$ dopamine $(10 \mathrm{mM}$ Tris buffer, $\mathrm{pH} 8.5$ ) at $37^{\circ} \mathrm{C}$ with the lid open to allow water to evaporate overnight. The substrates were sonicated 3 times, each time for $15 \mathrm{~min}$ in water to remove the weakly bonded dopamine. A second layer of polydopamine was obtained on repeating incubation, evaporation, and sonication. Then, five-layered polydopamine was obtained on repeating the abovementioned steps. L- or D-cystine was then further immobilized. A solution of $\mathrm{NaOH}$ with a concentration of $6 \mathrm{mM}$ was first prepared, and then, $\mathrm{L}$ - or D-cystine was added to obtain a final concentration of $3 \mathrm{mM}$. Next, the abovementioned polydopamine-grafted samples were incubated into the $\mathrm{L}^{-}$or D-cystine solution at $37^{\circ} \mathrm{C}$ for about $12 \mathrm{~h}$. Then, they were cleaned 3 times to remove the unbonded cystine.

The samples were referred as $\mathrm{Ti}-\mathrm{O}$ ( $\mathrm{Ti}-\mathrm{O}$ film), DOPA (5 layer polydopamine-grafted Ti-O film), L (L-cystine-immobilized on DOPA), and D (D-cystine-immobilized DOPA).

For the sample preparation for BSA preadsorption, $0.1 \mathrm{mg}$ $\mathrm{mL}^{-1}$ or $0.5 \mathrm{mg} \mathrm{mL} \mathrm{m}^{-1}$ BSA was used. After a 2 hour incubation, samples were washed 3 times and then dried at room temperature.

\subsection{Characterization}

XPS analysis was performed via a KRATOS XSAM800 X-ray photoelectron spectrometer using CuK $\alpha$. The pressure in the chamber was below $2 \times 10^{-9}$ Torr. The binding energy scale was referenced by setting $\mathrm{C}_{\mathrm{ls}}$ peak at $284.6 \mathrm{eV}$.

For water contact angle analysis, the samples were placed on the holder of a DSA100 drop shape analyzer. The contact angle of the drop on the surface was measured at room temperature. At least 6 contact angles on different areas were measured and averaged.

Quartz crystal microbalance with dissipation (QCM-D) (QSense AB, Sweden) was used to monitor the process of BSA adsorption. First, polydopamine was deposited onto the Aucoated single crystal sensor (10 $\mathrm{mm}$ diameter $\mathrm{Au}$ films). Subsequently, the polydopamine-deposited crystal sensors were inserted into the QCM-D instrument. PBS buffer was then pumped into the QCM-D channels to run base lines. After the base lines were stable, the $\mathrm{L}^{-}$and $\mathrm{D}^{-c y s t i n e}$ solutions were injected at a speed of $50 \mathrm{~L} \mathrm{~min}^{-1}$. When the QCM signals did not vary, PBS buffer was introduced into the channels again. Finally, when the signals were stable, BSA $\left(0.1 \mathrm{mg} \mathrm{mL}^{-1}\right)$ was injected into the channels followed by washing with PBS buffer.

\subsection{Nitric oxide detection}

NO release catalyzed by the samples was examined using Sievers 280i chemiluminescence analyzer (GE Co., U. S) ${ }^{17}$ Briefly, a 5 $\mathrm{mL}$ solution containing $130 \mu \mathrm{M}$ GSNO, $60 \mu \mathrm{M} \mathrm{GSH}$, and $500 \mu \mathrm{M}$ EDTA- $\mathrm{Na}_{2}$ (to chelate metal ion contaminates that might otherwise decompose RSNO) was added into the reaction vessel at $37^{\circ} \mathrm{C}$. The sample was hung above the solution until the detecting baseline was steady; then, the sample was pushed into the reaction vessel and the releasing NO was continuously purged from the test solution with nitrogen to the chemiluminescence analyzer.

NO produced by ECs was also detected by Sievers $280 \mathrm{i}$ chemiluminescence analyzer. Nitrite/nitrate derived from $\mathrm{NO}$ in the culture media could be reduced by vanadium trichloride $\left(\mathrm{VCl}_{3}\right)$, and then, the reduced NO could be directly detected. Sodium nitrite was used to obtain the standard curve. First, vanadium trichloride reduction solution was prepared as follows: $0.4 \mathrm{~g} \mathrm{VCl}_{3}$ was dissolved in water and $4 \mathrm{~mL} \mathrm{H}_{2} \mathrm{SO}_{4}$ was then added; after this, water was added to achieve constant volume of $50 \mathrm{~mL}$. Second, $4 \mathrm{~mL}$ vanadium chloride reduction solution was added into the reaction vessel under $90{ }^{\circ} \mathrm{C}$ water incubation and connected by a circulation cooling system. When the detecting baseline was steady at zero, $10 \mu \mathrm{L}$ ECs culture media was added by microsyringe. At last, the reduced NO was continuously purged from the test solution with nitrogen to the chemiluminescence analyzer.

\subsection{In vitro platelet adhesion test}

Fresh whole blood was centrifuged at $1500 \mathrm{rpm}$ for $15 \mathrm{~min}$ to separate the blood corpuscles and obtain platelet rich plasma (PRP). Due to its chemical instability, only a few endogenous donors of $S$-nitrosothiol can be preserved in the final PRP. 
Therefore, GSNO was added for compensation. For each sample, $0.5 \mathrm{~mL}$ of PRP was added, supplemented solutions of GSNO and GSH were subsequently added, and finally $130 \mu \mathrm{M}$ GSNO and $60 \mu \mathrm{M}$ GSH were obtained. Then, the samples were incubated in the PRP (with GSNO, GSH) for $30 \mathrm{~min}$. All the samples were tested in triplicate. The PRP was later removed and the samples were washed twice with PBS. These samples were treated with $2 \%$ glutaraldehyde for $12 \mathrm{~h}$, followed by a dehydration process. ${ }^{19}$ Fluorescence analysis was carried out via rhodamine-phalloidin dyeing.

\subsection{EC culture}

Human umbilical cords were donated by the Maternal and Child Care Service Centre of Chengdu. Human umbilical vein ECs (HUVECs) were isolated from new born umbilical cord according to Jaffe et al. ${ }^{20}$ using enzymatic digestion. Following isolation, HUVECs were cultured in M199 media (Gibco, U.S.A.) with 15\% FBS (Sigma, U.S.). Cells were cultured in humidified air containing $5 \% \mathrm{CO}_{2}$ at $37{ }^{\circ} \mathrm{C}$, and the fourth passage was used. ECs were seeded at a concentration of $5 \times 10^{4}$ cells per $\mathrm{mL}$ and incubated at $37^{\circ} \mathrm{C}$ in $500 \mu \mathrm{L}$ of M199 culture media with $15 \%$ and $20 \mu \mathrm{g} \mathrm{mL}{ }^{-1}$ endothelial cell growth supplement. For the NO release group, similar to the platelet adhesion test, GSNO and GSH were added to obtain a final concentration of $130 \mu \mathrm{M}$ and $60 \mu \mathrm{M}$, respectively. Culture media was changed every 6 hours for the NO release group. After the predetermined time points ( 1 day and 3 day), samples were taken out, and the non-adhered cells were eliminated by PBS washing. Then, the adhered cells were fixed with $2.5 \%$ glutaraldehyde for $4 \mathrm{~h}$. After this, cells were rhodamine-phalloidin stained for $20 \mathrm{~min}$ and rinsed. Then, the samples were visualized via fluorescent microscopy (Zeiss, Germany). Cell viability analysis was carried out using a cell counting kit-8 (CCK-8).

The migration of ECs on different samples $(n=4)$ was carried out via a modified method according to Yang et al. ${ }^{21}$. Briefly, $1 \mathrm{~cm}$ $\times 3 \mathrm{~cm}$ Ti foil was folded into an L shape (Fig. 1a). One side of the $\mathrm{Ti}$ foil was incubated in dopamine solution to prepare a polydopamine coating. Then, the samples were refolded at the polydopamine edge (polydopamine coating has an obvious brown color). After L/D cystine was immobilized on the polydopamine coating, HUVECs were seeded only on another arm of the Ti surface. The seeded cell density was varied to $5 \times 10^{5}$ cells per mL. After being cultured for $6 \mathrm{~h}$, a confluent monolayer was formed and samples were turned upside down. Then, the migration of the cells on the modified arm continued for another 1 day. Moreover, GSNO and GSH were added to obtain a final concentration of $130 \mu \mathrm{M}$ and $60 \mu \mathrm{M}$ for the NO release group; the culture media was changed every 6 hours. After fixing and immunostaining, the samples were viewed via a fluorescence microscope, and the migration distances were obtained.

For detecting the function of ECs, cells were pretreated with NO for 2 hours in Boyden chamber as follows. ECs were seeded on 24-well plates with a concentration of $5 \times 10^{4}$ cells per $\mathrm{mL}$. After 1 day culture, Boyden chamber with a $3 \mu \mathrm{m}$ pore size was laid on the plate (Fig. 1b). Then, $1 \mathrm{~cm} \times 1 \mathrm{~cm}$ samples of $\mathrm{L}$ or D were laid upside down in the chamber. GSNO and GSH were a

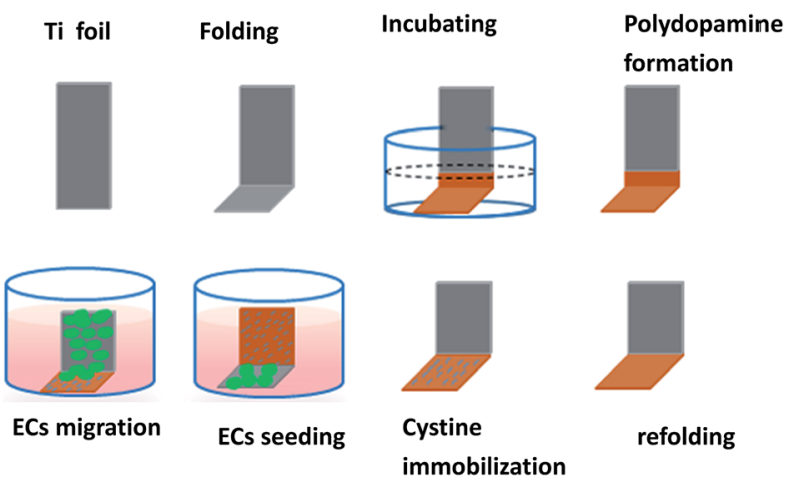

b

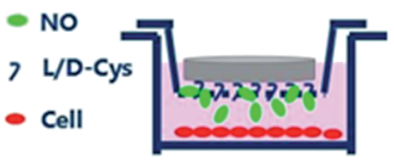

Fig. 1 Schematic of ECs migration and NO pretreatment experiment. (a) ECs migration experiment; (b) NO pretreatment experiment. ECs cultured in the media with GSNO and GSH, and samples laid upside down in the Boyden chamber.

added to obtain a final concentration of $130 \mu \mathrm{M}$ and $60 \mu \mathrm{M}$ in the cell culture media. After 2 hours culture, Boyden chamber was removed and culture media was replaced with new media without endogenous NO donor solution. At the predetermined time points, new media was added to replace the old media. Then, the media was obtained for nitrite/nitrate analysis.

\subsection{Statistical analysis}

Data are represented as mean values \pm standard deviation. Statistical analysis was performed using SPSS one-way ANOVA. A $p$ value less than 0.05 was considered to be statistically significant.

\subsection{Live subject statement}

The authors state that all experiments were performed in compliance with the guideline 'Biomedical research ethics review method involving people' (China), and approved by the medical ethics committee of Southwest Jiaotong University. Informed consents were obtained from human participants for this study.

\section{Results and discussion}

\subsection{Surface characterization}

Fig. 2a shows the XPS spectra of each sample. It was observed that after polydopamine deposition, no peaks of Ti2p or Ti2s appeared for the sample DOPA, indicating that the deposited polydopamine was thicker than the detection range of XPS. Additionally, a new peak of N1s that originated from the amino groups of dopamine molecules was present, proving that DOPA film was successfully coated onto the Ti-O surface. Additional peaks of S2s and S2p appeared for the sample $\mathrm{L}$ and D, which were due to the disulfide group of cystine. The surface chemical 
a

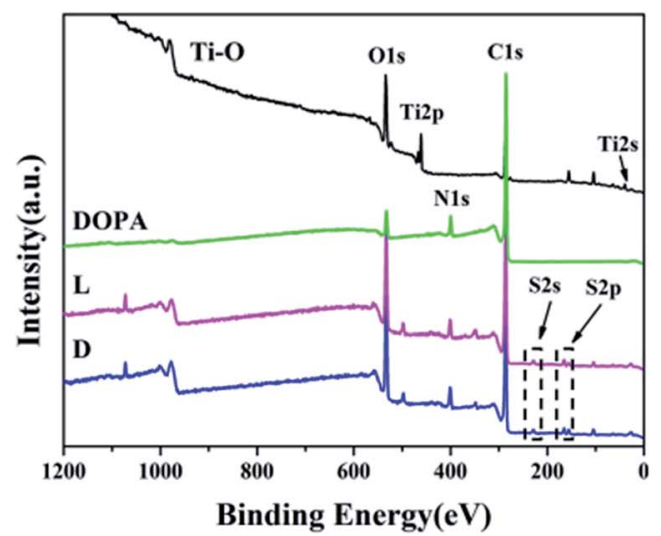

b

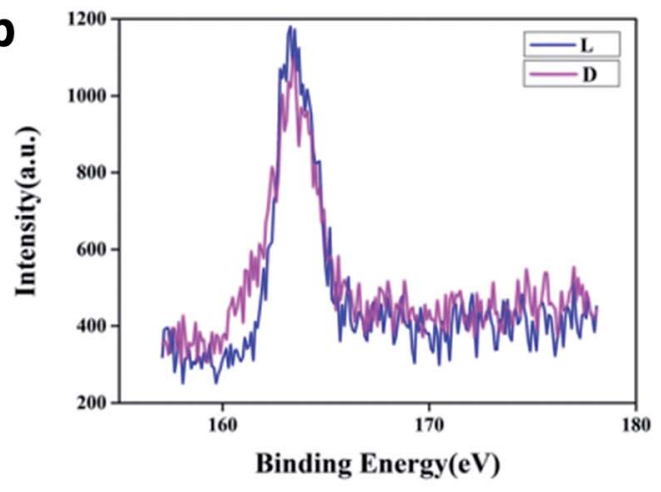

Fig. 2 XPS spectra of samples. (a) Wide-scan spectra and (b) high-resolution spectra of S2p on the sample $L$ and $D$.

compositions of samples L, D, and DOPA are listed in Table 1, and detailed high-resolution data for S2p of samples $\mathrm{L}$ and $\mathrm{D}$ are shown in Fig. 2b. There was slight difference in the surface $S$ content between the samples $\mathrm{L}$ and $\mathrm{D}$, consistent with the fact that molecules with different chiralities often have similar chemical reactivity.

Water contact angle of the samples is shown in Fig. 3a. It was observed the sample DOPA was hydrophilic. Sample L and sample $\mathrm{D}$ were more hydrophobic with a water contact angle about $60^{\circ}$. This may be because when $\mathrm{L}^{-}$or D-cystine was immobilized on the surface, hydrophilic groups, such as quinone and catechol group, on the surface of the sample DOPA were consumed and hydrophobic groups, such as $\mathrm{CH}_{2}$, were exposed on the surface. There were nearly no differences between the

Table 1 Surface element ratio of sample DOPA, L and D

\begin{tabular}{lllll}
\hline Samples & $\mathrm{C}(\%)$ & $\mathrm{N}(\%)$ & $\mathrm{O}(\%)$ & $\mathrm{S}(\%)$ \\
\hline DOPA & 73.3 & 7.5 & 19.2 & 0 \\
L & 75.6 & 6.1 & 16.2 & 2.1 \\
D & 75.8 & 6.2 & 16.0 & 2.0
\end{tabular}

samples L and D, indicating that surface chirality had little effect on hydrophilicity. However, sample $\mathrm{L}$ was more hydrophilic than sample D after BSA adsorption (Fig. 3b). A distinct difference of BSA adsorption between samples $\mathrm{L}$ and $\mathrm{D}$ was observed and more BSA was adsorbed on sample $\mathrm{L}$ than on sample $\mathrm{D}$, as monitored by QCM (Fig. 4). Since BSA is a hydrophilic protein, ${ }^{22}$ more BSA adsorption probably contributes to the decrease in the water contact angle for sample $\mathrm{L}$. Endogenous NO donor GSNO was not used in the QCM adsorption detection because GSNO adsorption on these surfaces can lead to NO release, which interferes with QCM detection.

\subsection{Discrimination in catalyzing NO release on different chiral surfaces}

Chiral discrimination in response to homochiral biological systems has been frequently reported in the literature..$^{23,24}$ Herein, NO release catalyzed by samples with different surface chiralities was evaluated using a Sievers 280i chemiluminescence analyzer. A steady and continuous NO release was observed when the samples were pushed into endogenous NO donor solution (Fig. 5). There was no burst release. A a

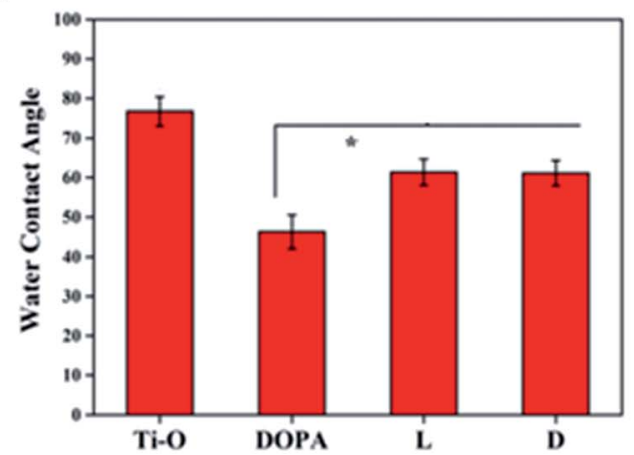

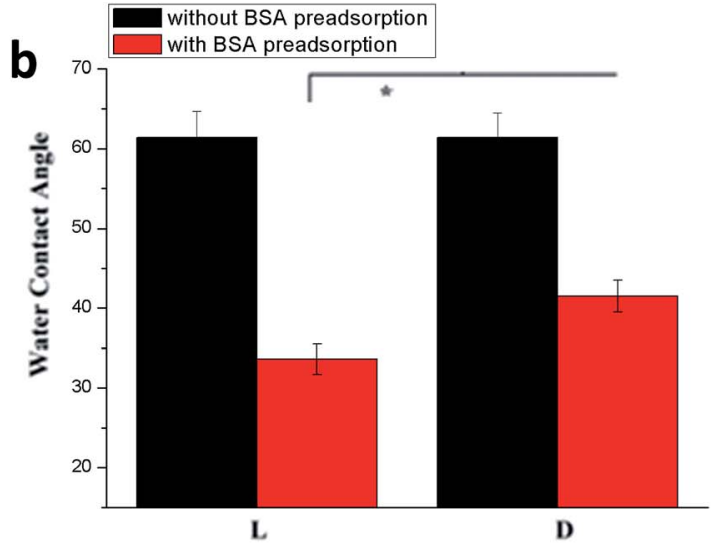

Fig. 3 Water contact angle of samples. (a) Bare samples; (b) sample L and D with or without BSA preadsorption $\left(0.1 \mathrm{mg} \mathrm{mL}^{-1}\right)$. Data are presented as mean $\pm \operatorname{SD}(n \geq 6, * p<0.05)$. 


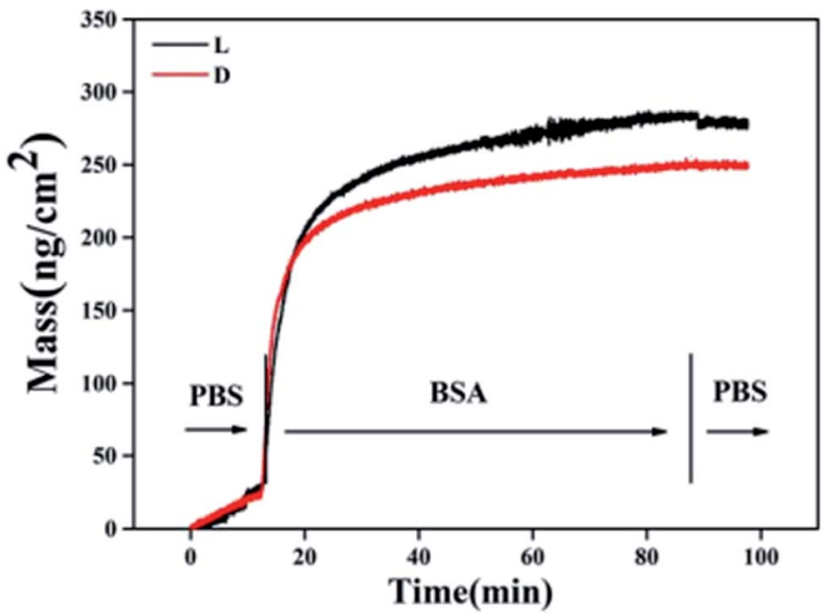

Fig. 4 QCM-D detection of BSA adsorption on sample $L$ and D (concentration of BSA: $0.1 \mathrm{mg} \mathrm{mL}^{-1}$ ).

significantly higher speed of NO release was observed when it was catalyzed by sample L compared to that by sample D. Results from the present study are in good agreement with previous studies, which show that surfaces immobilized with L-amino

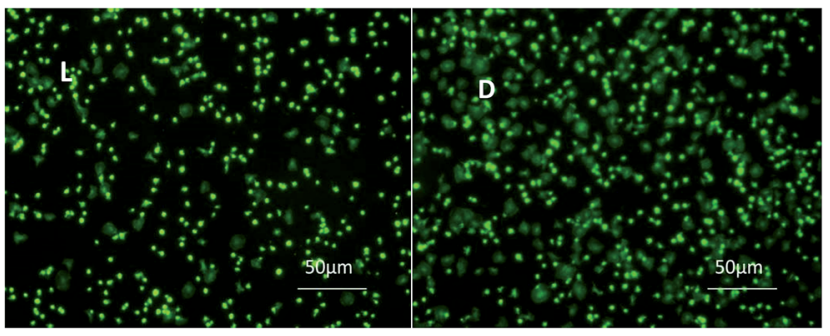

Fig. 6 Fluorescent images showing the platelet adhesion on $L$ and D sample after incubation with PRP and endogenous NO donor solution for $30 \mathrm{~min}$.

acid improve the protein adsorption regardless of the protein type. ${ }^{\mathbf{1 4 2 5}}$ Compared to sample $\mathrm{D}$, sample $\mathrm{L}$ also likely promotes the adsorption of GSNO, a nitro-derivative of GSH that is a protein fragment. Upon adsorption on the surface, GSNO is immediately consumed to produce NO, and thus, the process of GSNO adsorption converts into mass transfer. Distinct from other proteins' adsorption in equilibrium with desorption from a surface, GSNO is continuously transferred to the surface due to its constant consumption, which is driven by an intermolecular force similar to that in protein adsorption. Although a

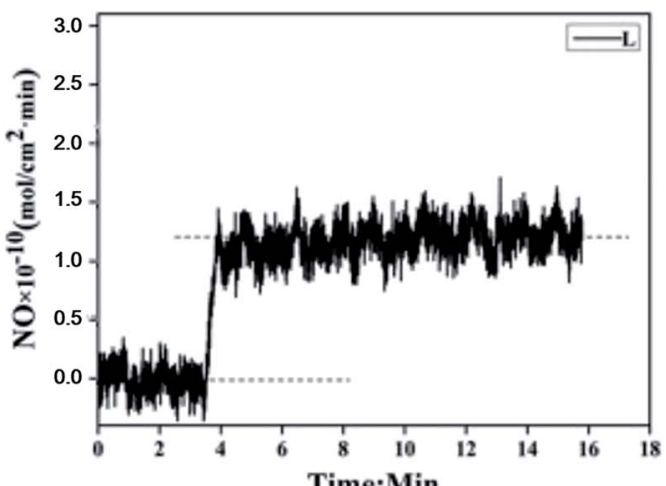

Time:Min

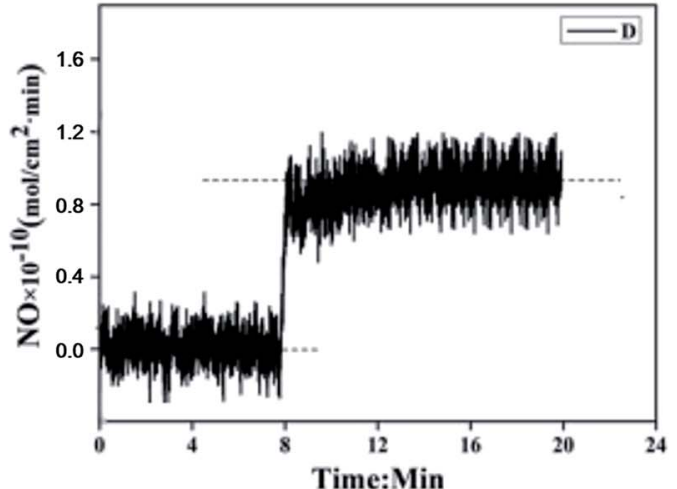

Time:Min

b

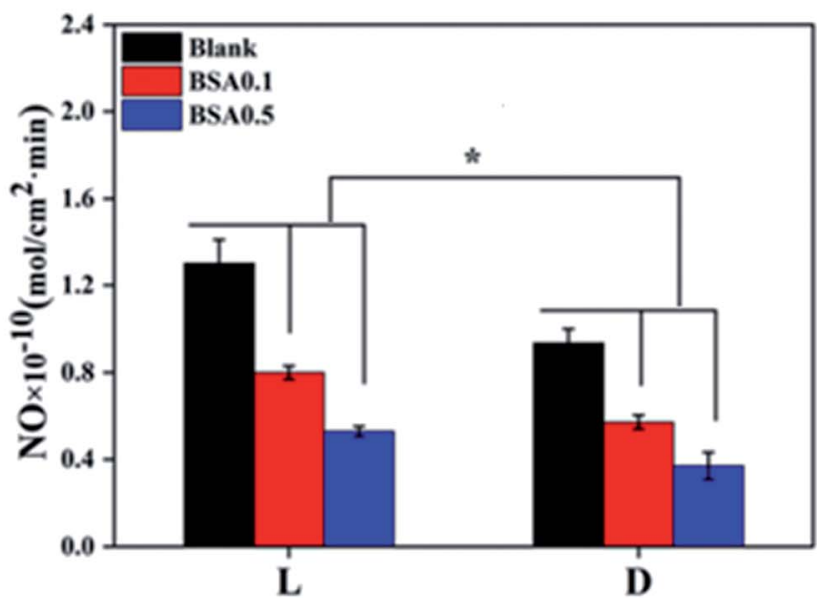

Fig. 5 NO release catalyzed by sample $L$ and D. (a) Monitored by Sievers $280 \mathrm{i}$ chemiluminescence analyzer. (b) NO release rates of samples with or without BSA preadsorption (BSA0.1 represents the concentration of BSA is $0.1 \mathrm{mg} \mathrm{mL}^{-1}$, BSA0.5 represents that of $0.5 \mathrm{mg} \mathrm{mL}{ }^{-1}$ ). Data are presented as mean \pm SD $(n \geq 4, * p<0.05)$. 
there was no difference in the surface S content or the amount of catalytic active sites between samples D and L, GSNO decomposition or NO release was enhanced with sample L due to faster transfer of GSNO.

When BSA was preadsorbed on the samples, NO release catalyzed by both samples decreased. Representative images are shown in the ESI (Fig. S1†). Despite more BSA adsorption, sample $\mathrm{L}$ retained higher catalyzing activity and induced more NO release under the same conditions. This may indicate that preadsorption of BSA on the sample $\mathrm{L}$ was reversible. Thus, when the samples were pushed into endogenous NO donor solution, desorption of BSA occured, whereas endogenous NO donor was adsorbed and then degraded to release NO. Thus, NO release mostly depended on the catalyzing activity of the samples. The abovementioned results illustrated that surface chirality had a significant influence on the catalyzing activity of the samples.

As a pivotal inhibitor of platelet activation, NO was loaded into biomaterials to enhance the hemocompatibility in peering studies. ${ }^{26,27}$ Biomaterials with higher NO release showed higher ability of platelet-activation inhibition. Platelet adhesion on samples $\mathrm{L}$ and $\mathrm{D}$ was evaluated, and the representative results are shown in Fig. 6 . The adhered platelets on sample L mostly remained round and only a few spread, indicating that less platelets were activated. In contrast, the spreading degree of adhered platelets on sample D was obviously increased, which was in accordance with the NO release results.

\subsection{Interactions of ECs with surface chemistry and NO}

It is of great importance to know how ECs interact with vascular biomaterials. Surface chemistry, including surface chirality, has profound effects on the cells. Even further, the synergetic effects of surface chirality combined with NO release on ECs remain unexplored. In the present study, ECs' adhesion, growth, and migration on each sample were studied. NO donor was then added in the culture media to explore the synergetic role of surface chemistry and NO release on ECs.

Fig. 7a shows ECs' adhesion and growth on the samples. Compared with DOPA, ECs preferred to adhere and proliferate on the L and D surfaces. Moreover, L surfaces presented better affinity for ECs according to CCK-8 analysis (Fig. 7b). Similarly, some previous studies show that surfaces modified by L-amino

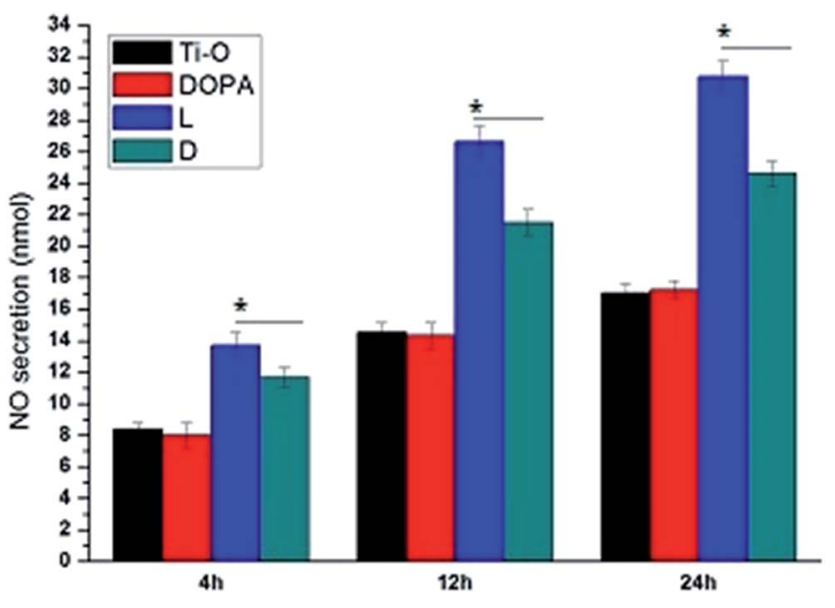

Fig. 8 NO secretion by ECs pretreated by $\mathrm{NO}$ donor solution and samples. Data are presented as mean $\pm \operatorname{SD}(n=4, * p<0.05)$. a
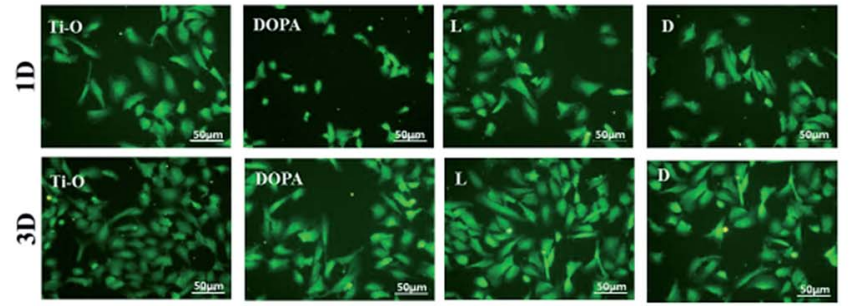

c
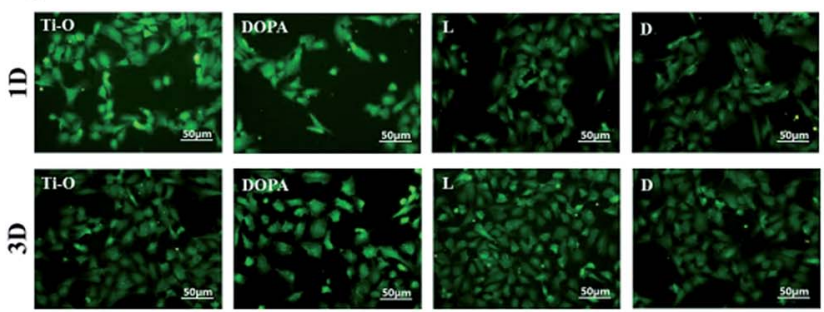

b

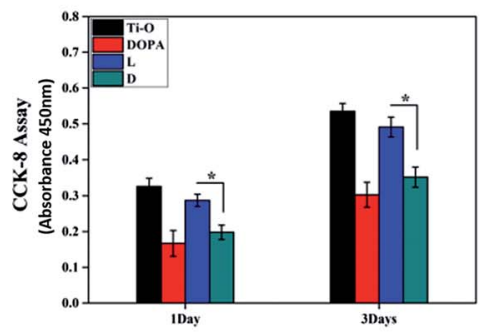

d

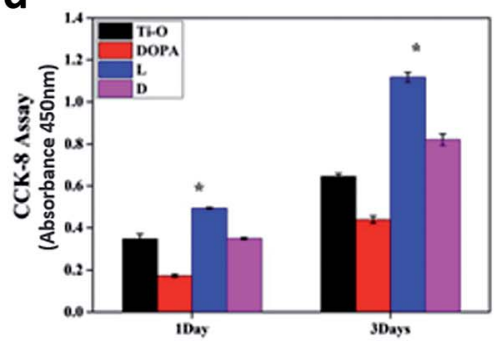

Fig. 7 Evaluation of ECs' adhesion, growth on samples and cell viability. (a) Fluorescent images of ECs adhesion and growth on samples for 1 day and 3 days respectively. (b) ECs' viability analyzed by CCK-8. (c) Fluorescent images of ECs adhesion and growth on samples for 1 day and 3 days with NO donor solution in media which was changed every 6 hours. (d) ECs' viability with NO donor solution which was changed every 6 hours in the media and analyzed by CCK-8. Data are presented as mean \pm SD $(n=4, * p<0.05)$. 
acid have better cell compatibility than those modified by $\mathrm{D}^{-}$ amino acid. ${ }^{25,28}$ Furthermore, Zhou et al. ${ }^{25}$ proposed that greater protein adsorption on L-amino acid-immobilized surfaces contributed to better cell compatibility. In the present study, we also found more protein (BSA) adsorption on the L surfaces.

When NO donor was added into the culture media, the spreading degree of adhered ECs decreased, especially on the $\mathrm{L}$ and D surfaces, compared to those without an added NO donor
(Fig. 7c). According to statistical analysis, there was a significant enhancement of cell viability on the $\mathrm{L}$ surfaces compared to that on Ti-O when NO donor was added (Fig. 7d). Additionally, cell viability on $\mathrm{D}$ surfaces recovered to a higher level than that on Ti-O after 3 days culture (Fig. 7d). Moreover, an obviously higher viability of ECs was retained on the $\mathrm{L}$ surfaces than that on the D surfaces. These results demonstrated that NO release increased the ECs' viability. Due to its short half life, most a
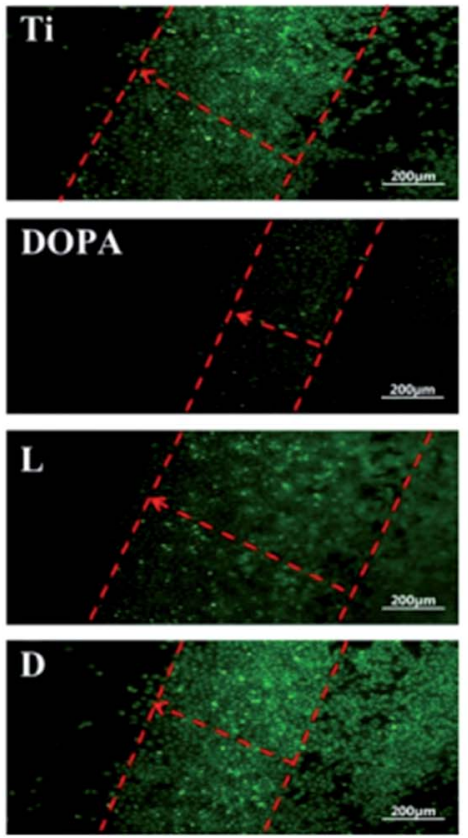

C
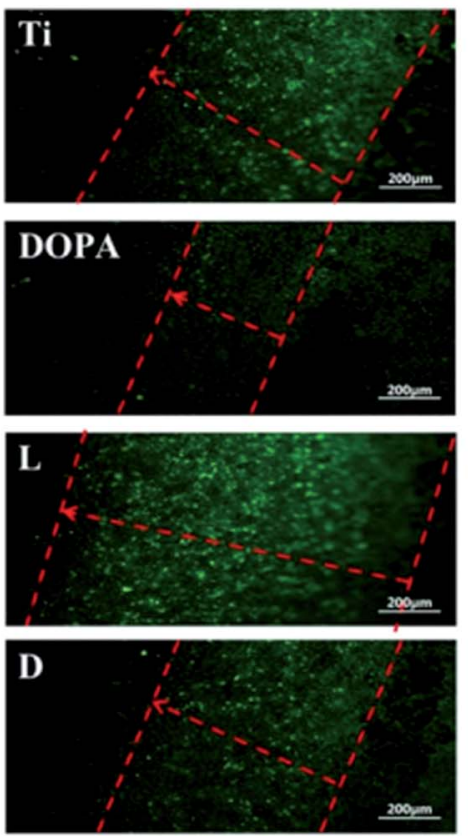

b

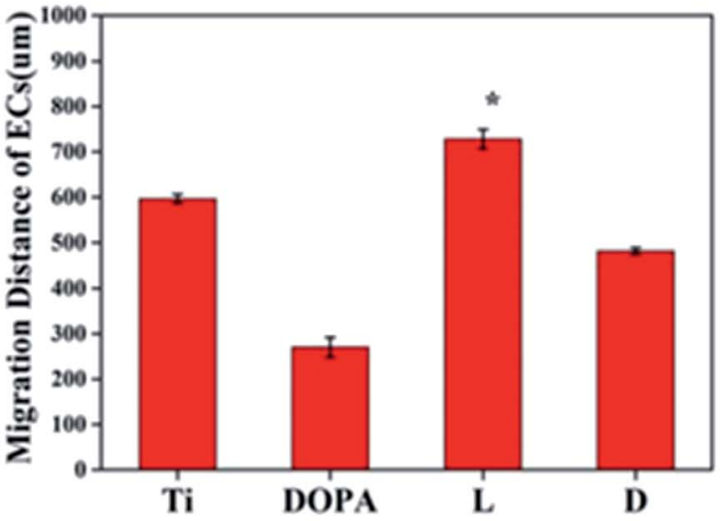

d

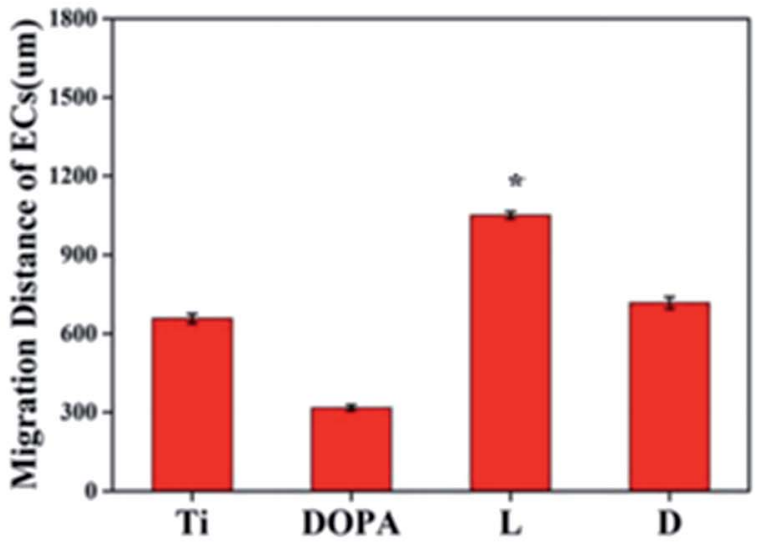

Fig. 9 Evaluation of ECs migration on samples. (a) Fluorescent images of ECs migration. (b) ECs migration distance measured. (c) Fluorescent images of ECs migration on samples with NO donor solution in the media, which was changed every 6 hours. (d) ECs migration distance measured with NO donor solution in the media, which was changed every 6 hours. Data are presented as mean \pm SD $\left(n=3,{ }^{*} p<0.05\right)$. 
researchers equate NO donor with NO. Sarkar et al. reported that various NO donors damaged ECs and stated that NO inhibited ECs' proliferation. ${ }^{11}$ They reported that NO donor undergoes self-decomposition by consumption of thiols. The consumption and generation of NO donor and thiols keep a balance (metabolism), and the concentration will remain constant in vivo. NO release via both catalyzation or selfdecomposition of NO donor will consume thiols. In their study, only NO donor was added, and thiols were consumed without supplying would change the microenvironment of oxidation and reduction. Interestingly, Law et $a .^{29}$ found that antioxidants protect ECs from NO donor damage. In addition, the self-decomposition of NO donor was uncontrollable, and NO release monitoring was not reported in their study. In our study, GSH was supplied with GSNO as the NO donor solution. Moreover, NO release was regulated by the catalytic activity of the samples. It was controlled to maintain a concentration of

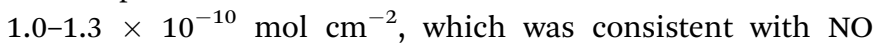
secretion by ECs.

To exclude surface chemistry influences, ECs were cultured in plates with NO donor-containing media and samples and pretreated for 2 hours (Fig. 1b). Following this, NO secretion by pretreated ECs was evaluated. Fig. 8 shows that there was no significant difference in the NO secretion of ECs between that pretreated with Ti-O and DOPA, and NO secretion significantly increased when the ECs were pretreated with L or D sample. Furthermore, greater NO secretion was shown by L sample pretreatment than D sample pretreatment. These results clearly showed more NO secretion of ECs with biomaterial-induced NO release. Previously, Chen et al. reported that endothelial nitric oxide synthase gene expression and activity were regulated by a positive-feedback regulatory action of exogenous NO. ${ }^{30}$ Increase in the activity of nitric oxide synthase may contribute to more NO secretion by ECs.

Both EC adhesion and migration are required for in situ endothelialization of vascular prostheses. The results, as shown in Fig. 9a and b, indicated the DOPA surfaces substantially reduced the migration of ECs, whose migration distance was only 0.5 times on Ti surfaces. Polydopamine is widely used for surface modification due to its high reactivity. High reactivity without selectivity of polydopamine may contribute to the low viability and mobility of ECs. Moreover, note that the $\mathrm{L}$ surface remarkably enhanced the mobility of the ECs, and the migration distance was 0.3 times and 0.6 times longer than that of $\mathrm{Ti}$ and D, respectively. When endogenous NO donor solution was added, EC migration on D surfaces recovered to 1.0 times that of the Ti surface, demonstrating the positive effect of NO release. ECs' migration on the L surface remained 0.6 times longer than that on the D surfaces. The abovementioned results confirmed that the combined effect of surface chemistry and NO release promotes the migration of ECs on sample $\mathrm{L}$.

\section{Conclusion}

In summary, we have reported that NO release and ECs' behavior on cystine-immobilized surfaces are highly dependent on the surface chirality. L surfaces promote more protein adsorption and higher NO release. Synergetic effects of surface chirality and NO release by L-cystine immobilization promote ECs' viability and migration on the surfaces. These results convincingly show that NO release and surface chirality regulated by L-cystine immobilization are efficient methods for anticoagulation and endothelialization, which appear promising for vascular devices modification.

\section{Acknowledgements}

The financial support for this work provided by the National Natural Science Foundation of China (No. 31270020), Sichuan Province Science Foundation for Youths (No. 2013JQ0043) and Fundamental Research Funds for the Central Universities (2682016YXZT14, 2682014CX006) is greatly acknowledged.

\section{References}

1 R. Priora, A. Margaritis, S. Frosali, L. Coppo, D. Summa, et al., In vitro inhibition of human and rat platelets by NO donors, nitrosoglutathione, sodium nitroprusside and SIN1, through activation of cGMP-independent pathways, Pharmacol. Res., 2011, 64, 289-297.

2 A. Kobsar, S. Simonis, E. Klinker, A. Koessler, S. Kuhn, et al., Specific inhibitory effects of the NO donor MAHMA/ NONOate on human platelets, Eur. J. Pharmacol., 2014, 735, 169-176.

3 B. Olas, Gasomediators ( ${ }^{\circ} \mathrm{NO}, \mathrm{CO}$, and $\mathrm{H}_{2} \mathrm{~S}$ ) and their role in hemostasis and thrombosis, Clin. Chim. Acta, 2015, 445, 115121.

4 T. C. Major, H. Handa, E. J. Brisbois, M. M. Reynolds, G. M. Annich, et al., The mediation of platelet quiescence by NO-releasing polymers via cGMP-induced serine 239 phosphorylation of vasodilator-stimulated phosphoprotein, Biomaterials, 2013, 34, 8086-8096.

5 Q. Zhao, J. Zhang, L. Song, Q. Ji, Y. Yao, et al. Polysaccharidebased biomaterials with on-demand nitric oxide releasing property regulated by enzyme catalysis, Biomaterials, 2013, 34, 8450-8458.

6 Y. Zhou, Y. Weng, L. Zhang, F. Jing, N. Huang, et al. Cystamine immobilization on $\mathrm{TiO}_{2}$ film surfaces and the influence on inhibition of collagen-induced platelet activation, Appl. Surf. Sci., 2011, 258, 1776-1783.

7 Y. Weng, Q. Song, Y. Zhou, L. Zhang, J. Wang, et al. Immobilization of selenocystamine on $\mathrm{TiO}_{2}$ surfaces for in situ catalytic generation of nitric oxide and potential application in intravascular stents, Biomaterials, 2011, 32, 1253-1263.

8 Z. Yang, Y. Yang, K. Xiong, X. Li, P. Qi, Q. Tu, F. Jing, Y. Weng, J. Wang and N. Huang, Nitric oxide producing coating mimicking endothelium function for multifunctional vascular stents, Biomaterials, 2015, 63, 8092.

9 N. Kang-Decker, S. Cao, S. Chatterjee, J. Yao, L. J. Egan, D. Semela, D. Mukhopadhyay and V. Shah, Nitric oxide promotes endothelial cell survival signaling through $S$ - 
nitrosylation and activation of dynamin-2, J. Cell Sci., 2007, 120, 492-501.

10 A. Papapetropoulos, G. G. Cardeña, J. A. Madri and W. C. Sessa, Nitric Oxide Production Contributes to the Angiogenic Properties of Vascular Endothelial Growth Factor in Human Endothelial Cells, J. Clin. Invest., 1997, 100, 3131-3139.

11 R. Sarkar, R. Clinton Webb and J. C. Stanley, Nitric oxide inhibition of endothelial cell mitogenesis and proliferation, Surgery, 1995, 118, 274-279.

12 T. Waldow, W. Witt, E. Weber and K. Matschke, Nitric oxide donor-induced persistent inhibition of cell adhesion protein expression and NFB activation in endothelial cells, Nitric Oxide, 2006, 15, 103-113.

13 S. Kofler, T. Nickel and M. Weis, Role of cytokines in cardiovascular diseases: a focus on endothelial responses to inflammation, Clin. Sci., 2005, 108, 205-213.

14 X. Wang, H. Gan and T. Sun, Chiral Design for Polymeric Biointerface: The Infl uence of Surface Chirality on Protein Adsorption, Adv. Funct. Mater., 2011, 21, 3276-3281.

15 D. Bandyopadhyay, D. Prashar and Y.-Y. Luk, Anti-Fouling Chemistry of Chiral Monolayers: Enhancing Biofilm Resistance on Racemic Surface, Langmuir, 2011, 27, 61246131.

16 X. Yao, Y. Hu, B. Cao, R. Peng and J. Ding, Effects of surface molecular chirality on adhesion and differentiation of stem cells, Biomaterials, 2013, 34(36), 9001-9009.

17 Y. Fan, X. Pan, K. Wang, S. Wu, H. Han, P. Yang, R. Luo, H. Wang, N. Huang, W. Tan and Y. Weng, Influence of chirality on catalytic generation of nitric oxide and platelet behavior on selenocystine immobilized TiO2 films, Colloids Surf., B, 2016, 145, 122-129.

18 Y. J. Weng, F. Qi, N. Huang, J. Wang, J. Y. Cheng and Y. X. Leng, Photochemical immobilization of bovine serum albumin on Ti-O and evaluations in vitro and in vivo, Appl. Surf. Sci., 2008, 255, 489-493.

19 R. Luo, L. Tang, J. Wang, Y. Zhao, Q. Tu, Y. Weng, R. Shen and N. Huang, Improved immobilization of biomolecules to quinone-rich polydopamine for efficient surface functionalization, Colloids Surf., B, 2013, 106, 66-73.

20 E. A. Jaffe, R. L. Nachman, C. G. Becker and C. R. Minick, Culture of Human Endothelial Cells Derived from Umbilical Veins, J. Clin. Invest., 1973, 52(11), 2745-2756.
21 Z. Yang, Q. Tu, M. F. Maitz, S. Zhou, J. Wang and N. Huang, Direct thrombin inhibitor-bivalirudin functionalized plasma polymerized allylamine coating for improved biocompatibility of vascular devices, Biomaterials, 2012, 33, 7959-7971.

22 Q. Wei, B. Li, N. Yi, B. Su, Z. Yin, F. Zhang, J. Li and C. Zhao, Improving the blood compatibility of material surfaces via biomolecule-immobilized mussel-inspired coatings, $J$. Biomed. Mater. Res., Part A, 2011, 96, 38-45.

23 K. Baranes, H. Moshe, N. Alon, S. Schwartz and O. Shefi, Neuronal growth on $\mathrm{L}^{-}$and D-cysteine self-assembled monolayers reveals neuronal chiral sensitivity, ACS Chem. Neurosci., 2014, 5(5), 370-376.

24 J. Deng, S. Wu, M. Yao and C. Gao, Surface-anchored poly(acryloyl L(D)-valine) with enhanced chirality-selective effect on cellular uptake of gold nanoparticles, Scientific Reports, 2016, 6, 31595.

25 F. Zhou, L. Yuan, D. Li, H. Huang, T. Sun, et al. Cell adhesion on chiral surface: The role of protein adsorption, Colloids Surf., B, 2012, 90, 97-101.

26 E. J. Brisbois, T. C. Major, M. J. Goudie, R. H. Bartlett, M. E. Meyerhoff and H. Handa, Improved hemocompatibility of silicone rubber extracorporeal tubing via solvent swelling-impregnation of $S$-nitroso- $N$ acetylpenicillamine (SNAP) and evaluation in rabbit thrombogenicity model, Acta Biomater., 2016, 37, 111-119.

27 T. C. Major, H. Handa, G. M. Annich and R. H. Bartlett, Development and hemocompatibility testing of nitric oxide releasing polymers using a rabbit model of thrombogenicity, J. Biomater. Appl., 2014, 29, 479-501.

28 X. Wang, H. Gan, T. Sun, B. Su, H. Fuchs, D. Vestweber and S. Butz, Stereochemistry triggered differential cell behaviours on chiral polymer surfaces, Soft Matter, 2010, 6, 3851-3855.

29 A. Law, J. Wu, L. Zeng and T. Wu, Aortic endothelial cells damaged by a nitric oxide donor and protected by flavonoids, Life Science, 1999, 64, 199-204.

30 J. Chen, L. C. Berry, M. Tanner, M. Chang, R. Paul Myers and B. Meyrick, Nitric Oxide Donors Regulate Nitric Oxide Synthase in Bovine Pulmonary Artery Endothelium, J. Cell. Physiol., 2001, 186, 116-123. 